\title{
Measurement and Metaphysics
}

\author{
Peter J. Lewis
}

January 21, 2014

\section{Introduction}

It is a prima facie reasonable assumption that if a physical quantity is measurable, then it corresponds to a genuine physical property of the measured system. You can measure a person's mass because human beings have such a property. You can measure the average mass of a group of people because groups of people have such a collective property. And so on.

Now it would be truly surprising - miraculous, perhaps - if you could determine the average mass of a group of people by making measurements on just one of them. To ascribe such a statistical property to an individual looks like a category mistake. At first glance, protective measurements seem to pull off just such a miracle, determining for example the expectation value of position for an ensemble of particles via a measurement performed on one of them. The lesson we are supposed to draw, of course, is that expectation values are not statistical properties at all, despite their name. Rather than being an average over an ensemble of systems, the expectation value of position for a particle is a physical property of the individual system, and the wave function, as the bearer of these properties, is a physical entity (Aharonov, Anandan and Vaidman, 1993).

The protective measurement procedure has been challenged (Uffink, 1999; Gao, 2013; Uffink, 2013), but for present purposes I will assume that protective measurements exist, at least in principle, that are capable of revealing "statistical" properties like expectation values in a single measurement. My aim here is not to challenge the existence of such a physical procedure, but rather to explore the arguments that connect the existence of protective measurements with conclusions concerning the nature of physical reality. What 
protective measurements are supposed to show is that "epistemological" interpretations of the quantum state are untenable - that the wave function of a system must instead be interpreted "ontologically" (Aharonov, Anandan and Vaidman, 1993: 4617).

But what exactly are the epistemological and ontological interpretations contrasted here? There are at least two distinct possibilities. ${ }^{1}$ First, the epistemological interpretation could be identified with an empiricist attitude towards quantum mechanics in general - taking the theory as a recipe for generating the probabilities of measurement results. Here the contrast is with scientific realism, construed as the view that quantum mechanics is in some sense a true description of the physical world. However, Dickson (1995) has convincingly argued that protective measurement cannot decide between empiricism and realism about quantum mechanics, since protective measurement is entirely consistent with empiricism. ${ }^{2}$ Hence I set this construal of the argument aside.

Second, the contrast between the epistemological and ontological interpretations of the wave function could be construed within an overall scientific realist attitude towards quantum mechanics in terms of the distinction between a statistical description and a categorical description of the physical system in question. Under this construal, the ontological interpretation is that the wave function is a description of the properties of a single physical entity, whereas the epistemological interpretation is that the wave function is a description of the distribution of properties over an ensemble of similar physical systems.

Taken in this way, the argument in favor of the ontological interpretation is more interesting. At first glance, though, it is still somewhat puzzling, since the conclusion of the argument is already the standard position among realist interpretations of quantum mechanics, prior to any consideration of protective measurement. No-go theorems (e.g. Bell, 1964; Kochen and Specker, 1967) are taken to show that it is impossible to interpret the wave function as a statistical distribution of properties over an ensemble of similar systems. Interference phenomena seem to show that elements of the wave function

\footnotetext{
${ }^{1}$ Aharonov, Anandan and Vaidman take the target of their argument to be the position that "the wave function represents at least partially our knowledge of the system" (1993: 4617), but this isn't decisive between the two construals I outline here.

${ }^{2}$ Dickson does contend, though, that protective measurement puts realism and empiricism "back on an even footing", since it counteracts the support for instrumentalism provided by ordinary impulsive measurement (1995: 135).
} 
physically interact with each other, and hence that the wave function is something like a physically real field. Indeed, all three of the major realist research programs in the foundations of quantum mechanics - Bohmian, Everettian and GRW - take the wave function to be a real dynamical entity. ${ }^{3}$

Perhaps the intention is just to put the last nail in the coffin of ensemble interpretations. However, my goal here is to show that even though ensemble interpretations face formidable (and well-known) obstacles, protective measurements don't lead to any additional difficulties. Rather, they provide us with a nice illustration of a conclusion for which we had considerable indirect evidence already, namely that quantum mechanics leads to a blurring of the distinction between the intrinsic properties of a system and the statistical properties of the ensemble of which it is a member. This conclusion goes for all realist interpretations of the quantum state, both the mainstream ones that take the wave function to be a real field and the more conjectural ones that take the wave function to describe our knowledge of an ensemble.

\section{Bohm's theory}

Although the usual target of arguments from protective measurement is ensemble interpretations of the wave function, protective measurements have also been used to argue against the tenability of Bohm's theory (Aharonov, Englert and Scully, 1999). The argument against Bohm's theory begins from a protective measurement that measures the wave function intensity in a small region around $x=0$ for a particle whose wave function is spread out along the $x$ axis. Wave function intensity, of course, is connected via the Born rule to the statistical properties of the system; in this case, it tells us the probability of finding the particle close to $x=0$ if we measure its position using a standard impulsive measurement. The fact that the wave function intensity itself can be directly measured seems to show that the wave function intensity is a physical property of the system.

By itself, this is no argument against Bohm's theory, since Bohm's theory already takes the wave function intensity to be a real physical property of the system; it is the wave function that pushes the particle around. Rather, the argument concerns the physical details of the measurement interaction. The protective measurement is effected by a Hamiltonian that links the state

\footnotetext{
${ }^{3}$ However, it is worth noting that some Bohmians argue that the wave function is a physical law rather than a physical entity (Dürr, Goldstein and Zanghì, 1996).
} 
of the measured particle with the state of a second particle that acts as a pointer. In particular, the Hamiltonian contains a weak, long-lasting coupling term in the region around $x=0$ between the two particles. This interaction induces a shift in the wave packet of the pointer particle proportional to the wave function intensity of the measured particle in the $x=0$ region.

What (supposedly) makes this interaction problematic for Bohm's theory is that the Bohmian particle configuration for the system never passes through the $x=0$ region. The interaction Hamiltonian is such that the measured particle and the pointer particle only interact within the $x=0$ region. So the Bohmian particle configuration for the system cannot represent the actual particle motions during a protective measurement. Protective measurements, so the argument goes, cannot be given a physically acceptable Bohmian analysis.

I think this argument involves a misunderstanding of the role of particles in the Bohmian theory. Bohmian particles are not dynamically active entities; they do not act on each other or on the wave function. Their role is passive and phenomenological; they are pushed around by the wave function and correspond to the observed outcomes of our measurements. So the fact that the particle configuration does not pass through the $x=0$ region is irrelevant to the explanation of the dynamical behavior of the system, since the particles are dynamically inert. All the action lies in the evolution of the wave function, construed as a real field. The wave function surely exists (in part) in the $x=0$ region, and the dynamical behavior of the wave function in this region, under the influence of the Hamiltonian, explains the motion of the wave packet for the pointer particle. The only role of the Bohmian particle configuration is to pick out one precise location in this wave packet as representing the observed outcome of the measurement. ${ }^{4}$

The confusion arises, I suspect, because the Hamiltonian itself is derived from an analogous interaction in classical mechanics, and in the classical case the interaction really does occur between particles at $x=0$. In classical mechanics, the particles are dynamically active - the locus of forces. Classically, if the particles did not pass through the interaction region, the interaction could not occur. The Bohmian particles take on the phenomenological role of the classical particles; their configuration corresponds to the observed out-

\footnotetext{
${ }^{4}$ Indeed, this minimal role for the particle configuration has been exploited by Everettians seeking to argue that the Bohmian particles are redundant (e.g. Brown and Wallace, 2005).
} 
come of a measurement. But they do not take on the dynamical role of the classical particles; that role is taken on by the wave function.

It is instructive to compare the protective measurement to standard twoslit interference, in which the particle passes through one slit or the other and the wave function passes through both slits. Suppose the Bohmian particle passes through the left-hand slit. Its trajectory is affected by whether or not the right-hand slit is open, even though the particle doesn't pass through the right-hand slit. One might try to parlay this into an objection to Bohm's theory - how could the particle be affected by the state of a slit it doesn't go near? - but clearly this would be unfair to the theory. The particle trajectory is not intended to be a dynamical explanation, as it is in classical mechanics.

This is not to say that Bohm's theory is unproblematic. In systems with more than one particle, the motion of one particle depends on the location of all the other particles, no matter how distant. This makes it hard to square Bohm's theory with special relativity, since the location of distant particles right now is undefined in special relativity. This problem certainly arises in protective measurements, since they involve more than one particle, but it is hardly a special problem for protective measurements, and it was well-known before protective measurements were postulated (e.g. Bell 1971).

\section{Contextual properties}

So Bohm's theory suffers from no special problem explaining protective measurements, but only generic problems of an explicitly non-local theory. And Bohm's theory is particularly useful in making explicit the distinction between contextual and non-contextual properties of quantum systems. Since this distinction will be important later, it is worth considering in detail how contextual properties arise in Bohm's theory.

A contextual property is one whose value depends on the context in which it is measured. The $x$-spin of a particle prepared in an eigenstate of $z$-spin provides a simple example of a contextual property in Bohm's theory. ${ }^{5}$ Suppose the $x$-spin of the particle is measured using a Stern-Gerlach device; if the particle is spin-up it is deflected upwards, and if it is spin-down it is deflected downwards. Since Bohm's theory is a deterministic theory, the possible trajectories of the Bohmian particles cannot cross in configuration space (as an intersection between trajectories would amount to indeterminism). In a

\footnotetext{
${ }^{5}$ My exposition here follows Albert (1992).
} 
single-particle experiment like that envisioned here, the configuration space is just ordinary three-space. This means that if the particle starts out in the upper half of the wave packet, it is deflected upwards, and if it starts out in the lower half it is deflected downwards (because otherwise the trajectories would cross). So (one might think), spin-up particles are just those that are located in the upper half of the wave packet.

But now suppose we rotate the Stern-Gerlach device by $180^{\circ}$ around the paths of the particles, so that spin-down particles are deflected upwards and spin-up particles are deflected downwards. Consider again a particle that starts out in the upper half of the wave packet; by the same argument as before, it must be deflected upwards. But now this particle is recorded as a spin-down particle. That is, a particle that starts out in the upper half of the wave packet will manifest itself as a spin-up particle if the Stern-Gerlach device is one way up, but a spin-down particle if the device it the other way up. The spin of the particle depends on the context in which it is measured.

This way of putting things makes it sound like spin in Bohm's theory is a property of particles, albeit of a peculiar kind. But this is somewhat misleading. In fact, rather than saying that spin is a (contextual) property of the measured system, it is more accurate to say that the measured system doesn't really have a spin property - that nothing in its pre-measurement state corresponds to the observed spin value. After all, the orientation of the Stern-Gerlach device can't affect the earlier state of the measured system, so prior to the measurement there can be no fact of the matter about whether the system has the spin-up property or the spin-down property. However, contextuality doesn't arise for a particle residing in a wave packet that is an eigenstate of $x$-spin. In such a case, the entire wave packet is deflected up (say), taking the particle with it, and if the Stern-Gerlach device is rotated, the entire wave packet is deflected down, again taking the particle with it. Here it does seem appropriate to ascribe a spin property to the system. And presumably the bearer of this spin property is the wave function, since as noted above, the particles are causally inert in Bohm's theory. So all in all, it seems that spin is a property that the wave function possesses in Bohm's theory, but only when the wave function takes certain forms (the spin eigenstates).

Similar considerations apply to the protective measurement described in the previous section. In this measurement, the wave packet of the measured particle is confined within a box centered on $x=0$ in the ground state (Aharonov, Englert and Scully, 1999: 138). The protective measurement 
effectively measures an observable for which this state is an eigenstate whose eigenvalue is the wave intensity around $x=0$ (Uffink 1999: 3479). The measurement outcome is recorded in the position of the pointer particle, which let us suppose moves along its $y$-axis.

But now suppose that the wave packet of the measured particle is initially a superposition of the ground state and the first excited state. This wave packet is not an eigenstate of the observable measured by the protective measurement; the ground state has large wave intensity around $x=0$, but the first excited state has small wave intensity here. (For this reason the measurement would not count as protective, since it disturbs the measured system.) Hence the the pointer particle ends up in a superposition of two distinct positions; its wave function has significant intensity in two distinct regions, close to $y=0$ and far from $y=0$. If the pointer wave function is initially concentrated close to $y=0$, then the wave function splits into two packets, one of which moves up and the other of which stays put. Consider the motion of Bohmian particles in this case. Since Bohmian trajectories cannot cross, if the particle starts in the upper half of the initial wave packet it moves up, and if it starts in the lower half it stays put. ${ }^{6}$ Now consider what happens if the direction of the measurement Hamiltonian is reversed; the term in the pointer superposition corresponding to the ground state moves down rather than up, so particles that start in the lower half of the initial wave packet move and those in the upper half stay put. For a given initial configuration of the Bohmian particles, then, whether the measurement indicates that the wave intensity close to $x=0$ is small or large depends on the orientation of the measuring device.

So just like $x$-spin, the property measured by this protective measurement is contextual according to Bohm's theory. And again, since the state of the measured system can't depend on measurements that have yet to be performed on it, to say that a property is contextual is tantamount to saying that it doesn't exist at all; the pre-measurement system lacks a property corresponding to wave intensity at the origin. As before, this contextuality doesn't arise when the state of the measured particle is an eigenstate of the

\footnotetext{
${ }^{6}$ Matters are complicated slightly here by the fact that we are dealing with a twoparticle system inhabiting a six-dimensional configuration space. Bohmian trajectories cannot cross in this configuration space. However, since the Bohmian trajectories are practically stationary in the coordinates of the measured particle (Aharonov, Englert and Scully, 1999: 144), this amounts to the condition that the trajectories do not cross in the coordinates of the pointer particle.
} 
measured observable (so that the measurement is genuinely protective). In that case, it is appropriate to ascribe a wave intensity property to the system, where the bearer of the property is the wave function rather than the particle. That is, the wave intensity measured by this protective measurement is a property that the wave function sometimes possesses - a property that it possesses when it is in the ground state, but does not possess when it is a superposition of the ground and first excited states.

One might object to this conclusion on the grounds that the measurement just described is not a protective measurement (as noted above), since it disturbs the measured system. A genuine protective measurement is available that measures the wave intensity around the origin for the superposition state; this requires a different Hamiltonian from to ensure that the measurement is non-disturbing. ${ }^{7}$ On the basis of this genuine protective measurement, one might reasonably infer that since the wave intensity can be measured in the superposition state, the wave function possesses a wave intensity property for this state too. However, it is equally true that one can measure the spin property of a particle in a superposition of distinct $x$-spin eigenstates without disturbing its state. Suppose the particle is initially in a symmetric superposition of $x$-spin eigenstates. Then one can measure the spin property of this particle without disturbing it by rotating the Stern-Gerlach device by $90^{\circ}$, since the symmetric superposition of $x$-spin eigenstates is an eigenstate of spin along the $z$-axis. But of course, although the particle has a $z$-spin property in this state, it does not have an $x$-spin property (and we know this because an $x$-spin measurement on the $z$-spin eigenstate is contextual).

A similar thing can be said in the case of wave intensity measurements. Although one can perform a non-disturbing measurement that returns a wave intensity value for the superposition state, the corresponding property that this state possesses cannot be the same as the property measured by the original protective measurement on the ground state. Whatever property the original protective measurement measures, the superposition of ground and protective states lacks it, since the measurement on this state is contextual. The superposition state has a related property, measured by the new

\footnotetext{
${ }^{7}$ It might be argued that the only difference in the Hamiltonian between the two measurements is the protective term that ensures that the measured state remains undisturbed. That is, the term in the Hamiltonian that defines the measured property remains unchanged. But it would need to be shown that a principled distinction can be drawn between these various parts of the Hamiltonian - one that does not beg the question by assuming that the measured property remains the same.
} 
protective measurement, one that perhaps has an equal claim to be called "wave intensity". Hence wave intensity, like spin, is best thought of as a family of related properties, not a single property, and different protective measurements measure different wave intensity properties.

The upshot of this argument is that although Bohmians typically take the wave function to be a physical entity, its property structure is quite complex. In particular, the intensity of the wave function around a particular point in (configuration) space is perfectly well-defined in the theory, but can generate disparate measurement results depending on how it is measured, indicating that it should not be viewed as a single possessed property of the system. That is, although the wave function underlies the physical properties of a system, in the sense that a difference in physical properties requires a difference in the wave function, it does not follow that all the mathematical properties of the wave function correspond to simple physical properties of the system. The contextual nature of wave intensity in Bohm's theory explored above suggests that even though wave intensity can be measured via a protective measurement, it should not be regarded as a simple physical property of the system.

\section{Ensemble interpretations}

In the previous section I argued that we have to be careful in our inference that the result of a protective measurement corresponds to a property of the measured system. So far, though, nothing I have said challenges the basic argument that protective measurements show that the wave function is a physical entity, and hence rule out ensemble interpretations of the wave function. Even though the wave intensity property can be contextual, there is no contextuality when the state is an eigenstate of the protective measurement operator. So the existence of protective measurements shows that there exist measurements that reveal "statistical" properties like wave intensity, and reveal them with certainty without disturbing the measured system. Hence we can turn Einstein's criterion of reality against him. Einstein famously held that "if, without in any way disturbing a system, we can predict with certainty (i.e., with probability equal to unity) the value of a physical quantity, then there exists an element of reality corresponding to that quantity" (Einstein, Podolsky and Rosen 1935, 777). Since protective measurements allow us to predict the values of wave function properties with certainty (and with- 
out disturbing the system), there must be elements of reality corresponding to these properties, undermining Einstein's own hope that the wave function can be interpreted as a statistical description of an ensemble rather than a physical entity.

As noted above, however, ensemble interpretations were apparently ruled out by the no-go theorems long before the advent of protective measurements. Still, there are those who continue to hold out hope. One prominent strategy for evading the no-go theorems is to invoke retrocausality - the hypothesis that causal influences can travel from later events to earlier events, as well as in the usual fashion from earlier events to later events (Cramer, 1986; Kastner, 2013; Price, 1994; Price and Wharton, 2013; Sutherland, 2008; Wharton, 2010). How does this strategy fare against the challenge of protective measurement?

It is important to note that contextuality is typically ubiquitous in retrocausal theories. Since causal influences can travel from later measurement events to earlier system preparation events, it is always possible for the initial state of a system to reflect the measurements that will later be performed on it. ${ }^{8}$ Unlike the contextuality that emerges in Bohm's theory, retrocausal contextuality can in principle even affect systems in eigenstates of the measured observable. For example, suppose that the state of a one-particle system is prepared in the spin-up eigenstate of $x$-spin. Then if the $x$-spin of the system will later be measured, the possessed properties of the system must correspond to $x$-spin-up, in the sense that those possessed properties will bring about the spin-up result with certainty. But if the $x$-spin of the particle will not later be measured, this fact can affect the earlier possessed properties of the particle, so they might differ from those that correspond to $x$-spin-up.

Consider what this means for the protective measurement of wave intensity discussed above. Like Bohm's theory, retrocausal theories ascribe determinate trajectories to a set of particles, but unlike Bohm's theory, there is no wave function steering the particles. So retrocausal theories cannot avail themselves of the response to Aharonov, Englert and Scully I gave on behalf of Bohm's theory; since there is no wave function in a retrocausal theory, the particles themselves must be the dynamically active entities. A further disanalogy with Bohm's theory is that we cannot rule out the possibility of particle trajectories crossing in the retrocausal case; since we have as

\footnotetext{
${ }^{8}$ Indeed, this is precisely how retrocausal theories evade the no-go theorems (Price, 1994).
} 
yet no explicit dynamics for a retrocausal theory, we cannot know whether a fully formulated retrocausal theory would be deterministic. But the crucial disanalogy with Bohm's theory is that any property can be contextual in a retrocausal theory, even the position of a particle, and even if the quantum state is an eigenstate of the observable to be measured.

Given this last point, it immediately follows that the property measured by a protective measurement can be contextual according to a retrocausal theory. That is, the possible particle trajectories can be sensitive to the kind of measurement that will be performed on the system. If a protective measurement is performed, this later fact can (in principle) cause the earlier particle trajectories to all pass through the region around $x=0$, and hence deflect the pointer particle via the interaction Hamiltonian. If on the other hand a standard impulsive measurement is performed on the system, this later fact can (in principle) cause the earlier particle trajectories to be statistically distributed according to the wave function intensity, so that the wave function can be interpreted epistemically. In the latter case, presumably only a small proportion of the particle trajectories pass through the region around $x=0$. (The caveat "in principle" is a huge one here, of course, since nobody has succeeded in explicitly constructing such a theory.)

What should we make of the ontological status of contextual properties in retrocausal theories? In Bohm's theory, I suggested that contextual properties are strictly speaking not properties of the system at all, since the state of the system now cannot depend on the measurements that will be performed on it later. But in a retrocausal theory, the state of the system now can depend on the measurements that will be performed on it later, so there is no barrier to contextual properties being genuine possessed properties of the system. And since there is no wave function in a retrocausal theory, these contextual properties have to be possessed properties of the particles in the system.

There is something a little strange in this, perhaps: the "wave intensity" property measured by the protective measurement is in fact the property of a particle, not a wave. But it is surely no stranger than "particle" properties like spin turning out to be properties of the wave function in Bohm's theory, GRW and Everett. In GRW and Everett "particles" are really just manifestations of the wave function; the wave function mimics particles under certain circumstances. Even in Bohm's theory, particle properties other than position are carried by the wave function. Retrocausal theories manifest the opposite effect; particles sometimes mimic the properties we might 
otherwise attribute to the wave function. The ontology underlying a particular measurement result depends on the theory used to explain that result; it can't be read off the measurement itself.

One might object that the fact that the particles in retrocausal theories have properties like "wave intensity" that can be measured using protective measurements means that the wave function in effect still exists, since wavelike properties are still physically instantiated. But it is worth noting that the contextuality of retrocausal theories means that only those "wave-like" properties whose values will be determined by protective measurements on the system are actually instantiated. A typical system subject only to impulsive measurements will have no such properties. A system subject to the above protective measurement will have a property corresponding to wave intensity at the origin, but not (for example) to the expectation value of position. In non-retrocausal theories like Bohm's theory the state cannot depend on which measurements will be performed on the system, and so all possible wave function properties must be instantiated at once. Only in this latter case must we postulate the wave function as a physical entity.

Of course, there are formidable obstacles to be faced by any ensemble interpretation of the quantum state. While retrocausal theories have long been proposed, nobody has yet succeeded at explicitly formulating the dynamics for such a theory. Perhaps it will prove to be impossible. My point here is only that protective measurements provide no new argument against ensemble interpretations; any interpretation that can bypasses the no-go theorems by appealing to contextual properties can thereby also evade the protective measurement argument for the reality of the wave function.

\section{Ensemble properties and individual prop- erties: a blurring of the lines}

Suppose an ensemble interpretation of quantum mechanics along the lines given in the previous section is possible. Then protective measurements can be explained without postulating a genuinely wave-like entity; the motion of the particle can fully explain the result. But there is still an air of mystery surrounding this account of protective measurement. The result of the protective measurement lines up with a genuinely statistical property - the proportion of particles one would find close to $x=0$ were you to perform a se- 
ries of ordinary impulsive measurements on an ensemble of similarly-prepared particles. What explains this agreement, if the protective measurement itself just records the value of a possessed property of the particle? How can the properties of a single particle reflect the statistical properties of an ensemble of particles?

What I wish to suggest is that this agreement is somewhat mysterious for all the major realist traditions for interpreting quantum mechanics, so there is nothing special about ensemble interpretations in this regard. The Bohmian, Everettian and GRW programs also explain the result of the protective measurement in terms of a possessed property of the system, albeit a property of the wave function. So these theories also face the problem of how a possessed property of an individual system can reflect the statistical properties of an ensemble of similarly-prepared systems. Postulating an entity that is spread out in configuration space as the bearer of the property does not solve the problem of how the actual wave-intensity property reflects the statistical properties of sets of unactualized impulsive measurements.

The mystery is deepest in Everettian quantum mechanics. There are no particles in the Everettian theory, so the result of the protective measurement described above is explained by a property of the wave function. What if an ordinary impulsive measurement is performed on the same system? Then the Schrödinger dynamics drives the wave function into a set of (practically) non-interacting branches, one for each possible outcome. The wave intensity of these branches matches the wave intensity of the corresponding elements of the original measured state - so, for example, if an impulsive measurement is performed to determine whether the particle is close to $x=0$, the intensity of the "yes" branch will be the same as the wave intensity of the measured state close to $x=0$. But now comes the tricky part: it needs to be established that the intensity of a branch corresponds to its probability. There as a well-known research program seeking to establish this (Deutsch, 1999; Wallace, 2012), but it remains controversial. There is certainly no obvious explanatory link between the intensity of a branch - which is a possessed property of a single entity - and the frequency of a given outcome in an ensemble. If DeutschWallace program succeeds, it does so by uncovering a surprising explanatory link here; if it does not succeed, it is because there is no such link at all.

Bohmian and GRW theories are built on the assumption that the Everettian explanation fails, and both attempt to add such an explanation by modifying quantum mechanics. The GRW collapse law is a stochastic law tailored specifically to connect wave intensity with the probability of recording the 
associated result were an ordinary impulsive measurement to be performed on the state. That is, the connection between the possessed properties of the wave function and the statistical properties of impulsive measurements is established by fiat. Whether one finds this genuinely explanatory probably depends on one's general feelings about the explanatory status of brute propensities (Dorato and Esfeld, 2010). Bohmian interpretations use much the same strategy. The Bohmian dynamical law is formulated specifically to ensure that the particle positions are always statistically distributed according to wave function intensity, hence connecting wave function intensity with the statistics of ordinary impulsive measurements in the required way. Again, this connection is established by fiat.

In this context, the explanation embodied by a putative ensemble interpretation does not look so bad after all. The explanation for the agreement between the result of the protective measurement and the statistical distribution of impulsive measurement results is that the retrocausal dynamics takes all the particle trajectories through the $x=0$ region in the protective case, but distributes them so that only a small proportion of them pass through this region in the impulsive case. Whether the dynamical law that accomplishes this shares the ad hoc flavor of the GRW and Bohmian dynamical laws remains to be seen; as yet we have no such law. It is promising, I think, that retrocausal theories are being developed based on the Feynman path construction, in which a quantum system probes all possible paths between two points (Wharton, Miller and Price, 2011). This makes it more plausible that a substantive explanation might be found whereby the present state of the system reflects the statistical properties of future possibilities. But even if no such explanation is forthcoming and the law establishes the link by fiat, it is in good company.

The main virtue of the literature on protective measurements, it seems to me, is to bring to the fore the rather remarkable connection between ensemble properties and individual properties in quantum mechanics. Prior to quantum mechanics, one would have said that applying a statistical property like an expectation value to an individual system is just a category mistake; statistical properties only properly apply to ensembles of identically-prepared systems. But the postulation of the wave function as a physical entity by Everettian, Bohmian and GRW quantum mechanics means that statistical properties like expectation values are reflected in the actual properties of a single system. Protective measurements do not provide a new argument for this conclusion, but they make it manifest in a remarkably direct way. 
But the link between the existence of protective measurements and the existence of the wave function as a physical entity should not be overstated. Some property of the measured system must correspond to the result of the protective measurement, but the protective measurement itself provides no evidence that the property must be instantiated by a wave-like entity rather than a particle. That is, measurement alone can't tell you what exists; rather, you have to look at the best theoretical explanation of the measurement results, and infer what exists from the ontological commitments of that theory. It is true that wave function explanations are dominant at the moment, but they are far from problem-free, and retrocausal particle-only explanations remain a promising alternative.

\section{Acknowledgements}

I would like to thank Shan Gao and the participants in the Workshop in Epistemology and Philosophy of Science at the University of Miami for very helpful comments on an earlier draft of this chapter.

\section{References}

Aharonov, Y., Anandan, J. and Vaidman, L. (1993). Meaning of the wave function. Physical Review A, 47, 4616-4626.

Aharonov, Y., Englert, B. G. and Scully, M. O. (1999). Protective measurements and Bohm trajectories. Physics Letters A, 263, 137-146.

Albert, D. Z. (1992). Quantum Mechanics and Experience. Cambridge, MA: Harvard University Press.

Bell, J. S. (1964). On the Einstein-Podolsky-Rosen paradox. Physics, 1, 195-200. Reprinted in Bell (1987).

(1971). Introduction to the hidden-variable question. Foundations

of Quantum Mechanics: Proceedings of he 49th International School of Physics "Enrico Fermi". New York: Academic, pp.171-181. Reprinted in Bell (1987).

(1987). Speakable and Unspeakable in Quantum Mechanics: Cambridge: Cambridge University Press. 
Brown, H. R. and Wallace, D. (2005). Solving the measurement problem: de Broglie-Bohm loses out to Everett. Foundations of Physics, 35, $517-540$.

Cramer, J. G. (1986). The transactional interpretation of quantum mechanics. Reviews of Modern Physics, 58, 647-687.

Deutsch, D. (1999). Quantum theory of probability and decisions. Proceedings of the Royal Society of London, A455, 3129-3137.

Dickson, M. (1995). An empirical reply to empiricism: protective measurement opens the door for quantum realism. Philosophy of Science, 62, $122-140$.

Dorato, M., and Esfeld, M. (2010). GRW as an ontology of dispositions. Studies in History and Philosophy of Modern Physics, 41, 41-49.

Dürr, D., Goldstein S. and Zanghì, N. (1996). Bohmian mechanics and the meaning of the wave function. In Experimental Metaphysics: Quantum Mechanical Studies in Honour of Abner Shimony, ed. R. S. Cohen, M. Horne and J. Stachel. Dordrecht: Kluwer, pp. 25-38.

Einstein, A., Podolsky, B., and Rosen, N. (1935). Can quantum-mechanical description of physical reality be considered complete? Physical review, 47, 777-780.

Gao, S. (2013). On Uffink's criticism of protective measurements. Studies In History and Philosophy of Modern Physics, 44, 513-518.

Kastner, R. E. (2013). The Transactional Interpretation of Quantum Mechanics. Cambridge: Cambridge University Press.

Kochen, S. and Specker, E. P. (1967). The problem of hidden variables in quantum mechanics. Journal of Mathematics and Mechanics, 17, 59-87.

Price, H. (1994). A neglected route to realism about quantum mechanics. Mind, 103, 303-336.

Price, H. and Wharton, K. (2013). Dispelling the quantum spooks - a clue that Einstein missed? arXiv:1307.7744. 
Sutherland, R. I. (2008). Causally symmetric Bohm model. Studies In History and Philosophy of Modern Physics, 39, 782-805.

Uffink, J. (1999). How to protect the interpretation of the wave function against protective measurements. Physical Review A, 60, 3474-3481.

(2013), Reply to Gao's "On Uffink's criticism of protective measurements". Studies In History and Philosophy of Modern Physics, 44, $519-523$.

Wallace, D. (2012). The emergent multiverse: quantum theory according to the Everett interpretation. Oxford: Oxford University Press.

Wharton, K. (2010). A novel interpretation of the Klein-Gordon equation. Foundations of Physics, 40, 313-332.

Wharton, K. B., Miller, D. J., and Price, H. (2011). Action duality: a constructive principle for quantum foundations. Symmetry, 3, 524540 . 